\title{
Effect of a Bombyx mori Protein Disulfide Isomerase on Production of Recombinant Antibacterial Peptides
}

\author{
Tae-Won Goo ${ }^{1}$, Seong-Wan Kim ${ }^{1}$, Kwang-Ho Choi ${ }^{1}$, Seong-Ryul Kim ${ }^{1}$, Seok-Woo Kang ${ }^{1}$, Seung-Won \\ Park $^{2}$, and Eun-Young Yun ${ }^{1 *}$ \\ ${ }^{I}$ Department of Agricultural Biology, National Academy of Agricultural Science, RDA, Suwon 441-100, Republic of Korea \\ ${ }^{2}$ Department of Biotechnology, Catholic University of Daegu, Daegu 712-702, Republic of Korea
}

\section{Abstract}

The insect baculovirus expression vector system (BEVS) is useful for producing biologically active recombinant proteins. However, the overexpression of heterologous proteins using this system often results in misfolded proteins and the formation of protein aggregates. To overcome this limitation, we developed a versatile baculovirus expression and secretion system using Bombyx mori protein disulfide isomerase (bPDI) as a fusion partner. bPDI gene fusion was found to improve the secretions and antibacterial activities of recombinant nuecin and enbocin proteins. Thus, we conclude that bPDI gene fusion is a useful addition to BEVS for the large-scale production of bioactive recombinant proteins.

Received : 22 May 2013

Accepted : 31 May 2013

\section{Keywords:}

Baculovirus Expression Vector System (BEVS),

Bombyx mori,

Antibacterial peptide,

Protein Disulfide Isomerase (PDI)

\section{Introduction}

The production of recombinant proteins from cultured insect cells for use in diagnostics, protein and biomedical research, and as vaccines is increasing, and currently is a major biotechnology research topic (Harrison and Jarvis, 2006; van Oers, 2006). The baculovirus expression vector system (BEVS) is a powerful recombinant protein expression system based on insect cells, which allow posttranslational modifications to occur in the endoplasmic reticulum (ER) (Yun et al., 2005a; Yun et al., 2005b). However, recombinant proteins obtained using the BEVS system often have low bioactivities and are poorly secreted, because of protein aggregation and degradation in the ER lumen. Endogenous protein biosynthesis in BEVS host cells is essentially shutdown immediately after viral infection because of high levels of exogenous gene production.
Moreover, this shutdown leads to molecular chaperone and foldase insufficiencies in the ER, which in turn result in protein aggregation (Fath-Goodin et al., 2006; Teng et al., 2013).

Protein disulfide isomerase (PDI) catalyzes the oxidation of disulfides and the isomerization of incorrect disulfides in new polypeptides during folding in the oxidizing environment of the ER. PDI consists of four domains (a-b-b'-a'); the a and a' domains contain catalytic CGHC motifs, whereas the $b$ and $b^{\prime}$ domains have no catalytic activity (Turano et al., 2002; Wilkinson and Gilbert, 2004). We previously isolated Bombyx mori protein disulfide isomerase (bPDI), which has two thiol oxidoreductase sites and enzymatic activity for reduced and scrambled RNase, like other PDI family members (Goo et al., 2002; Goo et al., 2008). However, bPDI expression is low in baculovirus-infected cells, especially when the recombinant protein is first expressed. In addition, is possible that PDI exhibits chaperone-like activity, which suppresses

\section{*Corresponding Author :}

Eun Young Yun

Department of Agricultural Biology, National Academy of Agricultural Science, RDA, Suwon 441-100, South Korea.

Tel: +82-31-290-8576 / FAX: +82-31-290-8543

E-mail: yuney@korea.kr 
aggregation, and thus, increases heterologous protein folding and secretion. We previously expressed the B. mori antibacterial peptide in insect Sf9 cells using BEVS, but failed to obtained high expression (Yun et al., 1997; Yun et al., 2002). For the large-scale production of bioactive antibacterial peptides, we constructed a modified BEVS vector that contains nuecin and enbocin fused with the bPDI gene. This modification was found to improve recombinant nuecin and enbocin secretion and antibacterial activity, which suggests that bPDI gene fusion can be used to improve the productions of other biologically active recombinant proteins.

\section{Materials and Methods}

\section{Experimental insect cell line}

Sf9 cells derived from the pupal ovarian tissue of Spodoptera frugiperda were maintained as an adherent cell culture in TC100 medium (Sigma) containing 10\% (v/v) heat-inactivated fetal bovine serum (FBS), as described previously (Yun et al., 2005a).

\section{Construction of transfer vector plasmid}

To construct secreted bPDI, the ER retention signal (KDEL; 5'AAA GAC GAG TTA-3') in the C terminus of bPDI cDNA was removed by double restriction enzyme digestion with BamHI and StuI. The resulting bPDI fragment was inserted into the pGEM-T vector to form pGEMT-(bPDI-KDEL). To construct the recombinant baculovirus, pGEMT-bPDI(-)KDEL was digested again with BamHI and StuI. The resulting bPDI-KDEL fragment was subcloned into the baculovirus vector $\mathrm{pBAC}-1$ to form pBAC1-(bPDI-KDEL). pBACl-(bPDI-KDEL), pBAC1nuecin, pBAC1-enbocin or pBAC1-(bPDI-KDEL)-nuecinenbocin was co-transfected with linearized viral DNA (Novagen) into $\mathrm{Sf} 9$ cells, and selection was performed by staining with 50 $\mathrm{mg} / \mathrm{ml}^{-1}$ neutral red and $250 \mathrm{mg} / \mathrm{ml}^{-1} \mathrm{X}$-Gal 3 days post-infection (p.i.). The plaques formed by the recombinant baculoviruses were plaque-purified three times and designated vAc-nuecin, vAc-enbocin or vAc-(bPDI-KDEL)-nuecin-enbocin.

\section{SDS-PAGE and Western blots}

Sf9 insect cells were mock-infected, or infected with wild- type Autographa californica nuclear polyhedrosis virus (AcNPV) or recombinant AcNPVs at a multiplicity of infection of $10^{6}$ in a $35-\mathrm{mm}$ dish. After incubation at $27^{\circ} \mathrm{C}$, cells were harvested $1,2,3,4$, or 5 days post-infection. For SDS-polyacrylamide gel electrophoresis (SDS-PAGE) of the cell lysates or media, uninfected Sf9 cells and virus-infected cells were washed twice with phosphate-buffered saline (PBS), mixed with protein sample buffer, and boiled. Total cellular lysates and media were then subjected to $12.5 \%(\mathrm{v} / \mathrm{v})$ SDS-PAGE. After electrophoresis, gels were fixed and stained with $0.1 \%$ (v/v) Coomassie Brilliant Blue R-250. Proteins were blotted onto polyvinylidene (PVDF) membranes (Amersham Biosciences) in transfer buffer [25 mM Tris- $\mathrm{HCl}, \mathrm{pH} 7.6$, and $192 \mathrm{mM}$ glycine in 20\% (v/v) methanol] at $30 \mathrm{~V}$, overnight at $4^{\circ} \mathrm{C}$. Membranes were then blocked in $1 \%$ bovine serum albumin (BSA) for $2 \mathrm{~h}$ at room temperature, and incubated with anti-6 $\times$ His-tag (Invitrogen) anti-serum $(1: 1,000$ $\mathrm{v} / \mathrm{v})$ for $1 \mathrm{~h}$ at room temperature. After washing in TBST (10 $\mathrm{mM}$ Tris-HCl, pH 8.0, $100 \mathrm{mM} \mathrm{NaCl}$, and 0.05\% Tween 20), membranes were incubated with alkaline phosphatase-conjugated goat anti-mouse $\operatorname{IgG}(1: 10,000 \mathrm{v} / \mathrm{v}$; Clontech) for $30 \mathrm{~min}$ at room temperature. After repeated washing, substrate solution $(10 \mathrm{mM}$ Tris- $\mathrm{HCl}, \mathrm{pH}$ 8.0, $100 \mathrm{mM} \mathrm{NaCl}$, and $5 \mathrm{mM} \mathrm{MgCl}_{2}$ ) containing Nitro-Blue Tetrazolium and 5-bromo-4-chloroindoyl phosphate was added. The reaction was quenched with distilled water.

\section{Antibacterial assays of recombinant proteins}

Recombinant proteins were tested for antibacterial activity using the inhibition zone assay (Ponti et al., 1999). Briefly, bacterial strains in the logarithmic phase were grown on LB medium. Sf9 cell supernatants were collected at 4 days postinfection. Supernatants were concentrated and poured onto small paper disks ( $3 \mathrm{~mm}$ diameter, $1 \mathrm{~mm}$ deep) placed on thin agar in $\mathrm{LB}$ medium containing test bacteria, and then incubated at $37^{\circ} \mathrm{C}$ for $18 \mathrm{~h}$. Antibacterial activity was identified by the formation of clear zones around wells after incubation.

\section{Results and Discussion}

The ER contains molecular chaperones that optimize the folding and assembly of newly synthesized secretory or cytoplasmic proteins. However, little is known about the role of PDI. In a 

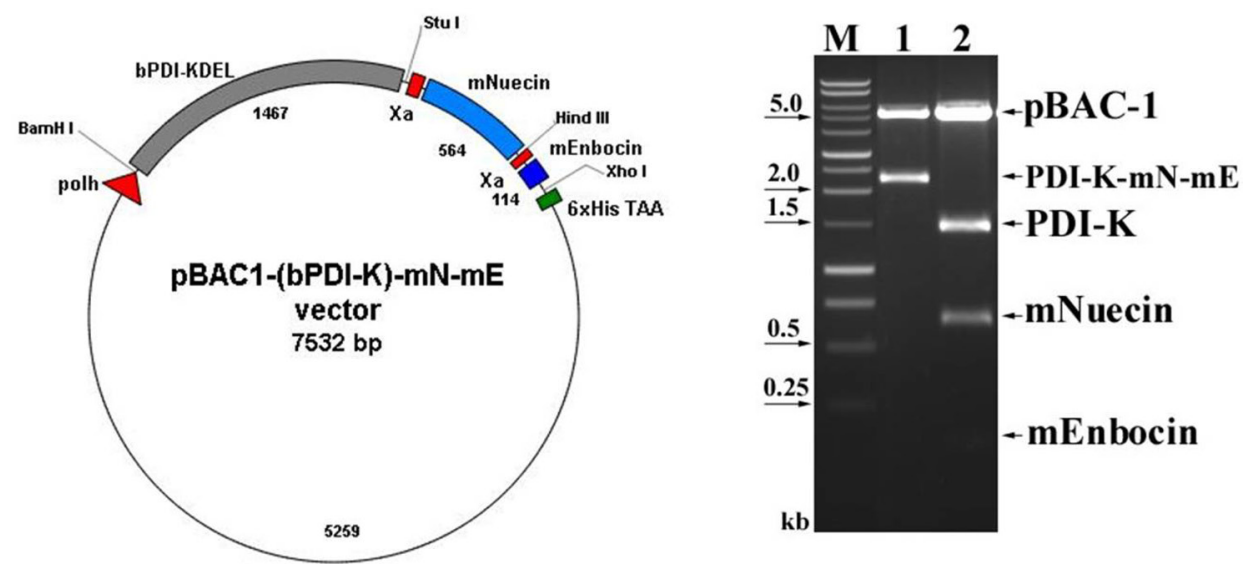

Fig. 1. Construction of baculovirus transfer vector for production of chemeric mature nuecin and enbocin fused bPDI lacking the ER retention signal KDEL. The open reading frame of bPDI lacking the ER retention signal KDEL (bPDI-KDEL) was subcloned into the BamHI and StuI site in baculovirus transfer vector pBAC-1. The coding sequence of mature nuecin (mNuecin) and enbocin (mEnbocin) linked factor Xa cleavage site was flanked by StuI and XhoI site, and then inserted into the pBAC1-(bPDI-KDEL) vector with StuI and XhoI. The baculovirus transfer vector pBAC1-(bPDI-KDEL)-mNuecin-mEnbocin was disgested with BamHI/XhoI (lane 1) and BamHI/ StuI/ HindIII/XhoI (lane 2). M, 1 kb ladder DNA markers.

previous study, we isolated and characterized bPDI (Goo et al., 2002), and because the over- or co-expression of chaperone proteins is known to enhance protein secretion, we attempted to increase secretory protein production by co-expressing bPDI in BEVS.

We previously showed that that removing the ER retention signal (KDEL) from bPDI cDNA increased bPDI secretion. We first removed the KDEL by digestion with Bam HI and Stu I, and then inserted the resulting bPDI fragment into the pGEM-T vector to form pGEMT-(bPDI-KDEL). After a second BamHI and StuI digestion, the bPDI-KDEL fragment was subcloned into the baculovirus transfection vector $\mathrm{pBAC}-1$ to form $\mathrm{pBAC} 1-$ (bPDI-KDEL), which contained an open reading frame for bPDI lacking the KDEL sequence. The coding sequence of mature nuecin (mNuecin) and enbocin (mEnbocin) linked factor Xa cleavage site was flanked by StuI and XhoI site, and then inserted into the pBAC1-(bPDI-KDEL) vector with Stu I and XhoI. The baculovirus transfer vector pBAC1-(bPDI-KDEL)mNuecin-mEnbocin was disgested with BamHI/XhoI (Fig. 1. lane 1) and BamHI/StuI/ HindIII/XhoI (Fig. 1. lane 2). We have confirmed that the (bPDI-KDEL)-mNuecin-mEmbocin was correctly inserted into pBAC-1 vector (Fig. 1). We then tested whether bPDI-KDEL improves the production of nuecin and enbocin by SDS-PAGE (Fig. 2A) and Westen blotting (Fig. 2B). Cells infected with vAc-(bPDI-KDEL)-nuecin-enbocin caused large amounts of nuecin and enbocin to be produced (Fig. 2A.

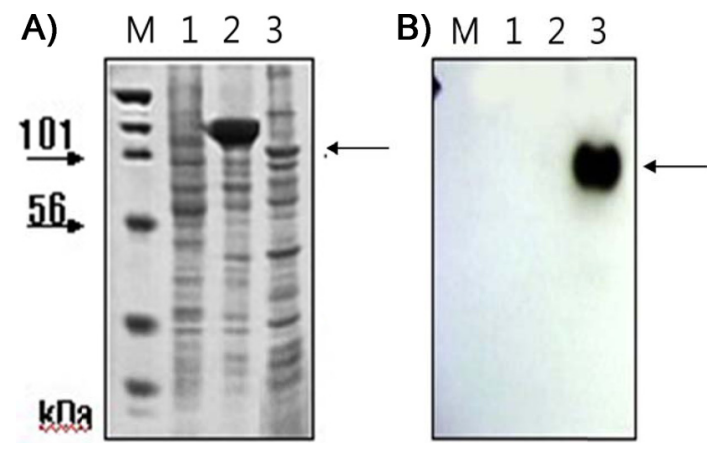

Fig. 2. SDS-PAGE of cell lysates (A) and Western blots of cell culture media (B) for chimeric nuecin and enbocin fused with bPDI-KDEL. Sf9 cells $\left(3.0 \times 10^{6}\right)$ were infected with recombinant baculovirus [vAc-(bPDI-KDEL)-mNuecin-mEnbocin; lane 3)] encoding (bPDI-KDEL)-mNuecin-mEnbocin-His6. Cells and cell culture media were harvested $96 \mathrm{~h}$ after infection (A). Western blots were performed using His6-tag antibody (B). Lane 1, proteins extracted from normal cells; lane 2, proteins extracted from cells infected with wild-type baculovirus. Arrows indicate the putative (bPDI-KDEL)-mNuecin-mEnbocin band.

lane 3, Fig. 2B. lane 3). Mis- or unfolded secretory proteins are known to be retained in the ER by ER chaperones like Bip and GRP94 (Kim et al., 1996). Thus, these results suggest that bPDI assists in the folding of newly synthesized poly-polypeptides for 


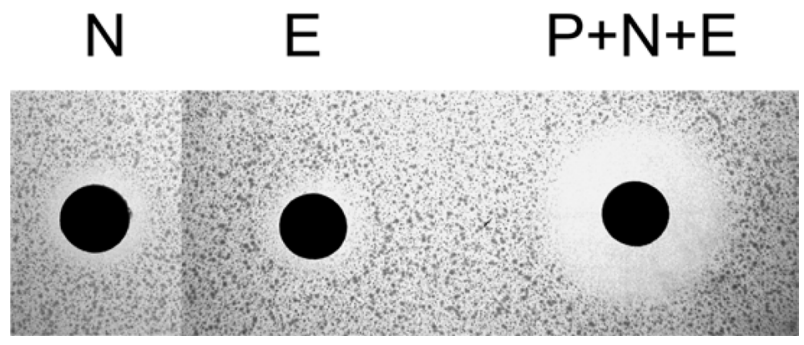

Fig. 3. Antibacterial activity of recombinant nuecin and enbocin protein against Escherichia coli. $5 \mathrm{ml}$ of culture medium $\left(2 \times 10^{6}\right.$ cells $\mathrm{ml}^{-1}$ ) were concentrated to $500 \mu \mathrm{l}$ using a freezing dryer; $40 \mu \mathrm{l}$ of concentrated sample were loaded onto a paper disk. N, recombinant nuecin; $\mathrm{E}$, recombinant enbocin; $\mathrm{P}+\mathrm{N}+\mathrm{E}$, chemric recombinant nuecin and enbocin with bPDI-KDEL.

oxidation and/or disulfide isomerization, which improves nuecin and enbocin production.

Nuecin and enbocin have antibacterial activity against Escherichia coli, but studies on the topic have been limited by poor nuecin production (Yun et al., 2002). To test the antibacterial activity of recombinant nuecin and enbocin, a supernatant containg the (bPDI-KDEL)-Xa-nuecin-Xa-enbocin fusion proteins were cleavage by the treatment of factor Xa protease, and then performed inhibition zone assays against $E$. coli (Fig. 3).

We performed inhibition zone assays against $E$. coli (Fig. $3)$. The antibacterial activity of nuecin and enbocin was approximately 24 -fold higher than that of nuecin or enbocin alone, indicating that bPDI promotes nuecin and enbocin trafficking and secretion and maintains nuecin activity. We also used inhibition zone assays to test the antibacterial activity of nuecin and enbocin produced against nine bacteria pathogenic in plants, i.e., Pseudomonas syringae, Pseudomonas tolaasii Staphylococcus aureus, Agrobacterium tumefaciens, Ralstonia solacerum, Erwinia mallotivora, Pectobacterium carotovorum, Erwinia chrysantuemi, and Bacillus megaterium (Fig. 4). Nuecin and enbocin showed the strongest activity against E. mallotivora, E. chrysantuemi, $R$. solacerum, and $P$. carotovorum. It showed moderate activity against $P$. syringae, $P$. tollasii, and $B$. megaterium, and weak activity against $A$. tumefaciens. However, Nuecin and enbocin was not ineffective against S. aureus (Fig. 4). These differences might be due to bacterial result from differences in the membrane potential differences of each bacterium.

The expression of exogenous proteins by BEVS often leads to protein aggregation and intracellular accumulation. Therefore, we

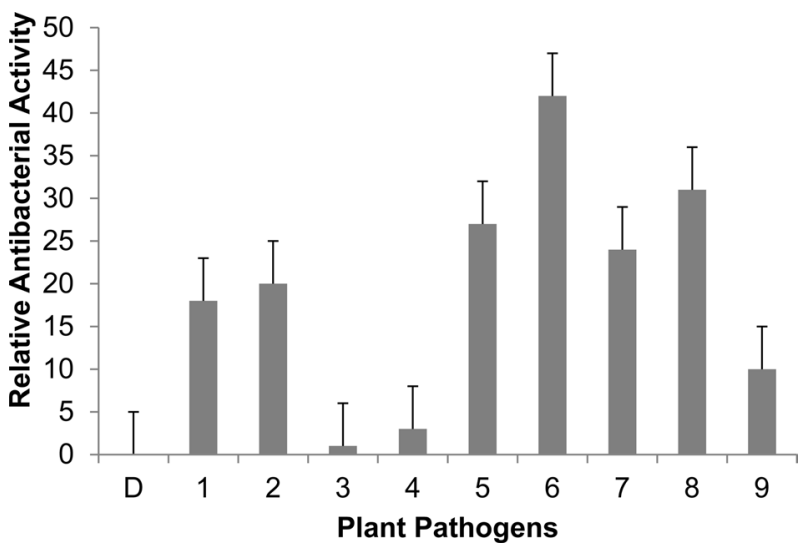

Fig. 4. Antibacterial activity of recombinant nuecin and enbocin against various plant pathogens. Five $\mathrm{ml}$ of culture medium $\left(2 \times 10^{6}\right.$ cells ml-1) were concentrated to $500 \mu \mathrm{l}$ using a freeze-dryer, and $40 \mu \mathrm{l}$ of concentrated samples were loaded onto paper disk. Values are the mean diameter of the clear zones \pm standard deviation from three independent experiments. $\mathrm{P}<0.05$ versus control. $\mathrm{D}$, distilled water (control); 1, P. syringae; 2, P. tollasii; 3, S. aureus; 4, A. tumefaciens; 5, R. solacerum; 6, E. mallotivora; 7, P. carotovorum; 8, E. chrysantuemi; and 9, B. megaterium.

developed a baculovirus expression and secretion system using bPDI as a gene fusion partner. Linking antibacterial peptides to bPDI was found to increase secretion and antibacterial activity, which suggests that bPDI may be useful for the mass productions of other recombinant proteins.

\section{Acknowlegements}

This work was supported by a grant from the Next-Generation BioGreen 21 Program (PJ009044032013), Rural Development Administration, Republic of Korea and by Bio-industry Technology Development Program (311059-4), Ministry for Food, Agriculture, Forestry and Fisheries, Republic of Korea.

\section{References}

Fath-Goodin A, Kroemer J, Martin S, Reeves K, Webb BA (2006) Polydnavirus genes that enhance the baculovirus expression vector system. Adv Virus Res 68, 75-90.

Goo TW, Yun EY, Hwang JS, Kang SW, Park S, You KH, Kwon OY 
(2002) Molecular characterization of a Bombyx mori protein disulfide isomerase (bPDI). Cell Stress Chaperones 7, 118-125.

Goo TW, Yun EY, Kim SW, Choi KH, Kang SW, Shin KS (2008)

Domain a' of Bombyx mori protein disulfide isomerase has chaperone activity. Z Naturforsch 63c, 435-439.

Harrison RL and Jarvis DL (2006) Protein N-glycosylation in the baculovirus-insect cell expression system and engineering of insect cells to produce "mammalianized" recombinant glycoproteins. Adv Virus Res 68, 159-191.

Kim PS, Kwon OY, Arvan P (1996) An endoplasmic reticulum storage disease causing congenital goiter with hypothyroidism. J Cell Biol $133,517-527$.

Ponti D, Mignogna G, Mangoni ML, De Biase D, Simmaco M, Barra D (1999) Expression and activity of cyclic and linear analogues of esculentin-1, an anti-microbial peptide from amphibian skin. Eur J Biochem 263, 921-927.

Teng CY, Chang SL, 3van Oers MM, Wu TY (2013) Enhanced protein secretion from insect cells by co-expression of the chaperone calreticulin and translation initiation factor eIF4E. Mol Biotechnol 54, 68-78.

Turano C, Coppari S, Altieri F, Ferraro A (2002) Proteins of the PDI family: unpredicted non-ER locations and functions. J Cell Physiol 193, 154-163.

van Oers MM (2006) Vaccines for viral and parasitic diseases produced with baculovirus vectors. Adv Virus Res 68, 193-253.

Wilkinson B and Gilbert H F (2004) Protein disulfide isomerase. Biochim Biophys Acta 1699, 35-44.

Yun EY, Goo TW, Hwang JS, Kim SH, Kang SW, Kim KY, Jin BR (2002) Expression of antibacterial protein, Nuecin, using baculorivus expression vector system in Bm5 insect cell and Bombyx mori. Kor J Seric Sci 44, 69-73.

Yun EY, Goo TW, Kim SW, Choi KH, Hwang JS, Kang SW, Kwon OY (2005a) Changes in cellular secretory processing during baculovirus infection. Biotechnol Lett 27, 1041-1045.

Yun EY, Goo TW, Kim SW, Choi KH, Hwang JS, Kang SW, Kwon OY (2005b) Galatosylation and sialylation of mammalian glycoproteins produced by baculovirus-mediated gene expression in insect cells. Biotechnol Lett 27, 1035-1039.

Yun EY, Kim SH, Kang SW, Jin BR, Kim KY, Kim HR, Han MS, Kang SK (1997) Molecular cloning and expression of the novel attacin-like antibacterial protein gene isolated from Bombyx mori. Kor J Appl Entomol. 36, 331-340. 